\title{
Structural, thermal and optical investigation of tin sulfide nanoparticles for next-generation photovoltaic applications
}

\author{
Nouman Rafiq ${ }^{1,2, *}$, WaqAR A.A. Syed ${ }^{1}$, Aulia Rifada ${ }^{1}$, M. Asad Ghufran ${ }^{3}$, \\ IJAZ-UR-REHMAN SHAH ${ }^{1}$, AHSAN Ali ${ }^{1}$, WIQAR HUSSAIN SHAH ${ }^{1}$ \\ ${ }^{1}$ Department of Physics, International Islamic University, Islamabad, Pakistan \\ ${ }^{2}$ Department of Material Science and Engineering, Tsinghua University Beijing, China \\ ${ }^{3}$ Department of Environment Sciences, International Islamic University, Islamabad, Pakistan
}

\begin{abstract}
We report a simple approach for synthesizing monodispersed, crystalline and size-tunable tin sulfide nanoparticles for environment friendly next generation solar cell applications. Both $\mathrm{SnS}$ and $\mathrm{SnS}_{2}$ nanoparticles could be a potential nanomaterial for solar cells. The structural, morphological, thermal and optical properties were investigated using X-ray diffraction (XRD), scanning electron microscopy (SEM), thermal gravimetric analysis (TGA), diffuse reflectance spectroscopy (DRS) and Fourier transform infrared spectroscopy (FT-IR). The XRD spectra revealed hexagonal and orthorhombic phases of $\mathrm{SnS}$ and $\mathrm{SnS}_{2}$ nanoparticles, respectively, where the grains size ranged from $11 \mathrm{~nm}$ to $30 \mathrm{~nm}$. The weight percentage as a function of temperature was determined using TGA analysis. Functional groups were observed by FT-IR. The energy bandgap was determined as $1.41 \mathrm{eV}$ showing usefulness of the nanoparticles in next generation environmental friendly solar energy applications.
\end{abstract}

Keywords: SnS nanoparticles; energy bandgap; next generation photovoltaics

\section{Introduction}

For the last few decades, II-VI compound semiconductors, particularly cadmium based materials, have been the subject of research owing to their wide range of applications in optoelectronic devices. However, these materials are not environment-friendly; some of them are poisonous and badly affect the human health and environment. The health risks associated with the inhalation of cadmium have been well documented [1,2]. The European legislation has introduced restrictions for the use of cadmium, particularly as a coating material for both the aerospace and the semiconductor industries [3]. This drove the researchers to work in the area of new environment-friendly semiconductors with suitable energy band gap. In this research, the attention has been given to the synthesis and characterization of tin-based chalcogenide semiconductors for the next generation environmental friendly solar material.

\footnotetext{
*E-mail: nouman.rafiq@iiu.edu.pk
}

In daily life, humans face a direct or indirect exposure to nanomaterials present in the variety of products and processes of natural and man-made nanostructured materials [4], which may cause cytotoxic effects, leading to various diseases in humans and animals [5]. Many of these are studied for their interactions with biological systems. However, tin sulfide is considered as intrinsic n-type semiconductor [6]. Tin sulfide has not been investigated thoroughly for its chemical, physical and toxicological properties. The sulfides of heavy metals are generally known to be insoluble, so it has a little toxic effect. Another important material having a potential for photovoltaic applications in the microelectronics industry is gallium arsenide, an inorganic arsenic compound that has impacted adversely on human health [7]. These arsenic compounds may cause irritation of skin and mucous membranes. One study found that the highly reactive surface of cadmium telluride quantum dots triggers extensive reactive oxygen damage to the human cell membrane. In addition, the cadmium telluride films are typically recrystallized in a toxic compound of cadmium chloride. 
Nanoparticles of narrow bandgap IV-VI compound semiconductors such as $\mathrm{SnTe}, \mathrm{PbS}$, and $\mathrm{SnS}$ are optically active in NIR and IR regions, and are of great interest for their applications in photovoltaics, IR detectors, and biomedical applications [8]. These applications have stimulated the search for a convenient synthetic methodology to make tin sulfide nanoparticles with narrow size distributions and well-defined optical properties.

Synthesis of mono-dispersed chalcogenide nanoparticles with cubic structures has attracted much attention due to its simplicity. SnS adopts an orthorhombic layered crystal structure, similar to distorted $\mathrm{NaCl}$ structure. The synthesis of uniform nanoparticles of IV-VI semiconductors with layered structures, small enough in the quantum confinement regime, is still a challenge for the researchers' community.

Cubic tin sulfide is a binary chalcogenide semiconductor that has recently attracted interest because it is considered as an excellent candidate for absorbing material for the development of nextgeneration environmentally friendly solar cells. Therefore, $\mathrm{SnS}$ nanoparticles $\left(\mathrm{E}_{\mathrm{g}}=1.1 \mathrm{eV}\right.$ to $1.4 \mathrm{eV}$ ) have a great potential as an absorber material [9-13] and in applications of solid state devices [14-17]. It has the P-type conductivity which can be controlled with a variety of dopants. Both tin and sulfur are cost-effective, safe to handle and non-pollutant for the environment. Electrical properties of $\mathrm{SnS}$ including carrier concentration and resistivity have been reported in the literature $[10,18,19]$. Ullah et al. [20] have numerically analyzed the performance of PV based devices on SnS.

It is, therefore, very important to have detailed work on the physical characteristics of $\mathrm{SnS}$ nanoparticles and their suitability for photonics and optoelectronic device fabrication. In this paper, an attempt has been made to prepare $\mathrm{SnS}$ nanoparticles using co-precipitation method and their physical properties have been investigated in terms of photovoltaic applications.

\section{Experimental}

\subsection{Synthesis of tin sulfide nanoparticles}

Tin sulfide nanoparticles were synthesized through wet chemical route with the following fundamental steps. The precursor materials were completely dissolved in deionized water and mixed in a flask for stirring till complete mixing. After few minutes, precipitates were formed, which were filtered and further washed to remove impurities. The flow chart for synthesis process is shown in Fig. 1. All the chemicals used in this work were of analytical grade with high purity. Tin chloride $\left(\mathrm{SnCl}_{2} \cdot 2 \mathrm{H}_{2} \mathrm{O}\right)$ and sodium sulfide $\left(\mathrm{Na}_{2} \mathrm{~S}\right)$ were taken as tin and sulfur precursors, respectively. 0.5 molar solutions of $\mathrm{SnCl}_{2}$ and $\mathrm{Na}_{2} \mathrm{~S}$ were prepared in two different flasks. Sodium sulfide solution was added dropwise into the tin solution with continuous stirring. The turning of colorless tin chloride solution into dark brown indicated the formation of $\mathrm{SnS}$ nanoparticles. The solution was stirred continuously with a magnetic stirrer for 2 hours. Then, the precipitated particles were further centrifuged at $400 \mathrm{rpm}$ for $5 \mathrm{~min}$ and washed several times with deionized water and ethanol. The final product was dried at $80{ }^{\circ} \mathrm{C}$ in the oven for 2 hours. Fig. 1 shows the fundamental steps for synthesis of nanoparticles.

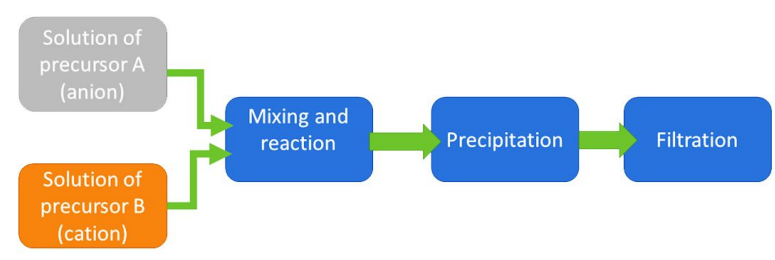

Fig. 1. Fundamental steps for synthesis of nanoparticles.

\section{Results and discussion}

\subsection{Structural analysis}

To examine the structural properties, the SnS nanoparticles have been characterized and evaluated through X-ray diffractometry (XRD). X'Pert Phillips diffractometer with $\mathrm{CuK} \alpha$ radiation at a wavelength of $1.5 \AA$ was used. Fig. 2a presents 
a diffraction pattern of $\mathrm{SnS}$ nanoparticles, which shows that the nanoparticles have a hexagonal structure, confirmed by the standard JCPDS Card No. 00-001-0984. The diffraction pattern shows the preferred orientations at (1 0101$)$ for tin-disulfide and $\left(\begin{array}{lll}0 & 2 & 1\end{array}\right)$ for tin mono-sulfide. The four prominent diffraction peaks ( $\left(\begin{array}{lll}0 & 2 & 1\end{array}\right),\left(\begin{array}{lll}1 & 3 & 1\end{array}\right),\left(\begin{array}{lll}2 & 0 & 0\end{array}\right)$ and $\left(\begin{array}{lll}2 & 2 & 1\end{array}\right)$ marked with circles, show the formation of hexagonal phase of SnS. Three diffraction peaks of the planes (llll $\left.0 \begin{array}{lll}0 & 2\end{array}\right),\left(\begin{array}{lll}1 & 0 & 1\end{array}\right)$ and $\left(\begin{array}{lll}1 & 1 & 1\end{array}\right)$ marked with squares clearly indicate the presence of orthorhombic phase of $\mathrm{SnS}_{2}$ and are confirmed by the standard JCPDS Card No. 01-083-1758. The pattern was fitted using X'Pert High Score software [21] and other lattice parameters were determined.

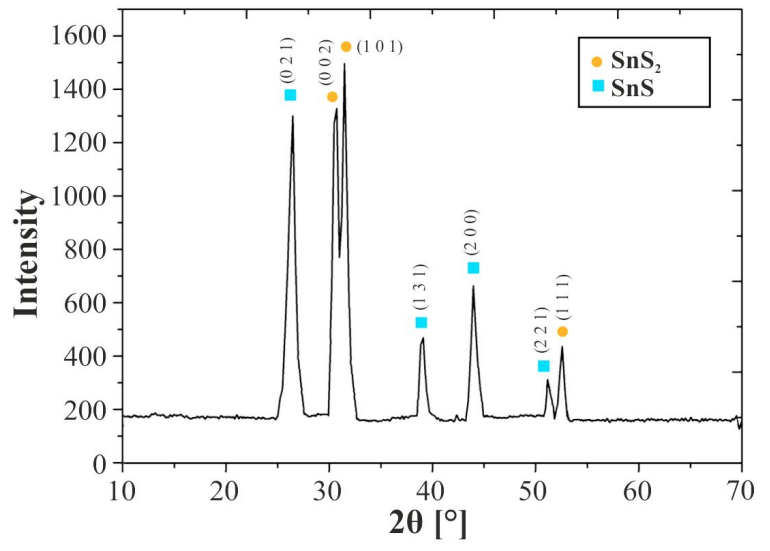

(a)

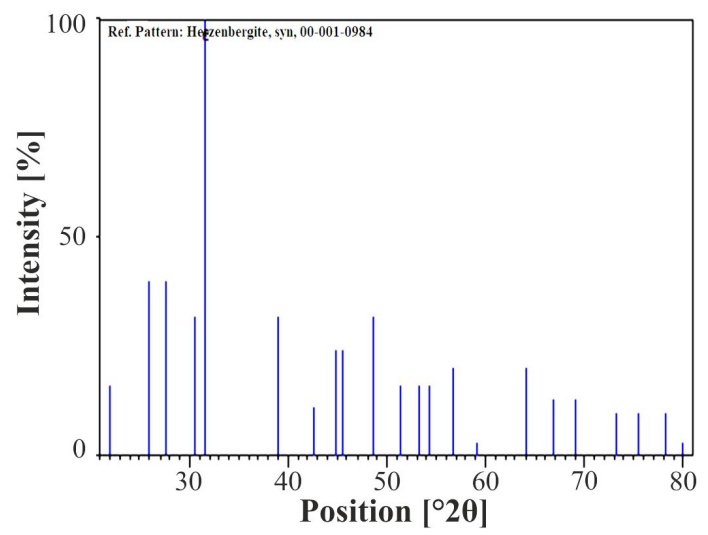

(b)

Fig. 2. XRD patterns of tin-sulfide nanoparticles: (a) diffraction pattern of tin-sulfide nanoparticles; (b) reference pattern.
The lattice parameter of the nanoparticles was determined by a well-known Bragg relation:

$$
n \lambda=2 d \sin \theta
$$

and the relation:

$$
\frac{1}{d^{2}}=\frac{h^{2}}{a^{2}}+\frac{k^{2}}{b^{2}}+\frac{l^{2}}{c^{2}}
$$

The interplanar spacing $\mathrm{d}$ of the planes was calculated as $1.337 \AA$ and the lattice constants as: $\mathrm{a}=\mathrm{b}=3.65 \AA, \mathrm{c}=5.60 \AA$. Using Scherer formula, the particle size was also estimated:

$$
\tau=\frac{k \lambda}{\beta \cos \theta}
$$

where the $\tau$ is particle size, $\theta$ is Bragg diffraction angle, $\lambda$ is $X$-ray wavelength and $\beta$ is full width at half maximum (FWHM) for the diffraction peak at $2 \theta$. The average particle size was measured as $22.1 \mathrm{~nm}$. All of these lattice parameters were calculated using X'Pert High Score software.

\subsection{Scanning electron microscopy}

SEM images have been taken to investigate the surface morphology of the obtained tin sulfide nanoparticles. The image in Fig. 3 shows nanometer size, uniformly distributed grains covering the whole surface. The particles are in aggregation state due to extremely small dimensions and high surface area. It is clearly shown that the nanoparticles are oval in shape with different sizes. The particle size distribution was evaluated by taking the average of size of these particles, which is $50 \mathrm{~nm}$ with an uncertainty of $\pm 10 \mathrm{~nm}$. Probably the variation in size is due to the synthesis conditions and therefore it is crucial to optimize the growth conditions to get the controlled size nanoparticles.

Fig. 4 shows energy dispersive spectroscopy (EDS) analysis of tin sulfide nanoparticles. The EDS analysis revealed the presence of $\mathrm{Sn}$ and $\mathrm{S}$, however sodium was also detected in the EDS study.

The atomic and the weight percentages of the elements, as observed in EDS spectra, are given in Table 1.

A significant amount of oxygen, chlorine and sodium was found in the EDS analysis. The oxygen was present in the air during the drying process. 


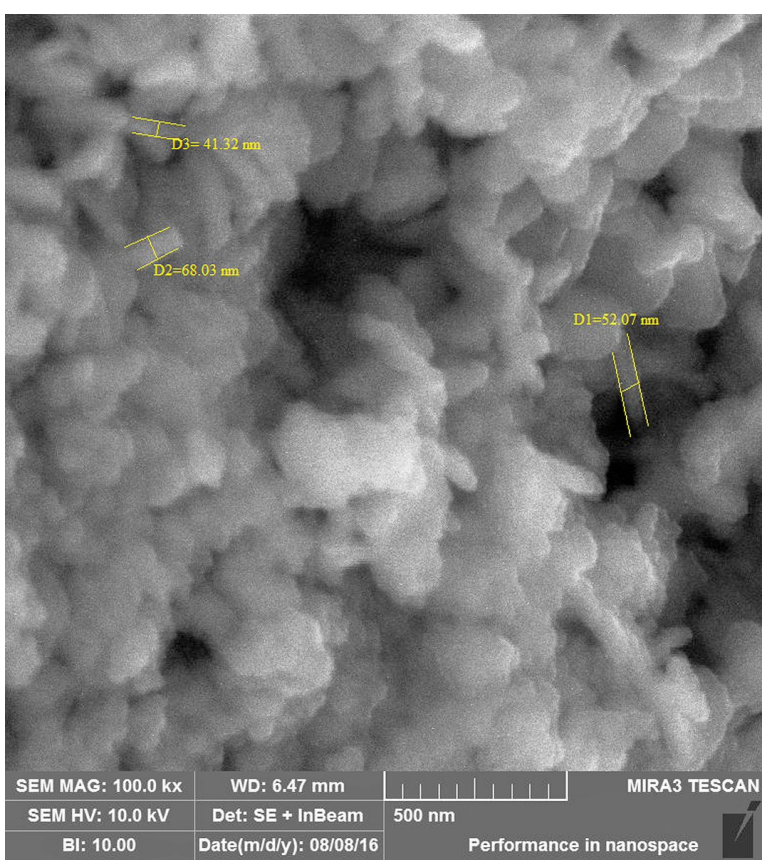

Fig. 3. SEM image of tin sulfide nanoparticles.

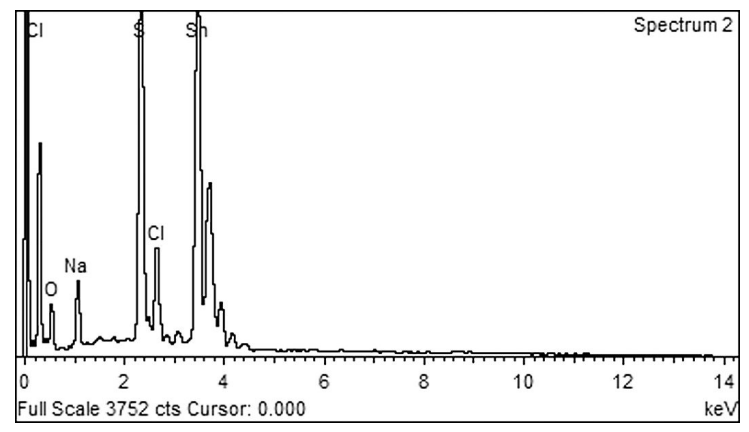

Fig. 4. Energy dispersive spectroscopic analysis of tin sulfide nanoparticles.

\subsection{Thermogravimetric analysis}

The weight of nanoparticles was monitored as a function of temperature and time by thermogravimetric analysis (TGA). TGA was used to analyze sample composition, thermal stability and kinetic parameters of the chemical reaction of nanoparticles. A small quantity of the sample was heated from $0{ }^{\circ} \mathrm{C}$ to $1000{ }^{\circ} \mathrm{C}$ with steps of $10{ }^{\circ} \mathrm{C} / \mathrm{min}$. The graph presented in Fig. 5 explains the thermal stability of the sample, where continuous decreasing in weight was observed by heating the sample up to $300{ }^{\circ} \mathrm{C}$. This decrease in weight was due to evaporation of water.
Table 1. Elemental composition revealed by EDS.

\begin{tabular}{lcc}
\hline Element & wt.\% & at.\% \\
\hline \hline sodium & 5.61 & 10.11 \\
chlorine & 5.37 & 6.27 \\
oxygen & 17.10 & 44.27 \\
sulfur & 15.10 & 19.51 \\
tin & 56.82 & 19.83 \\
\hline
\end{tabular}

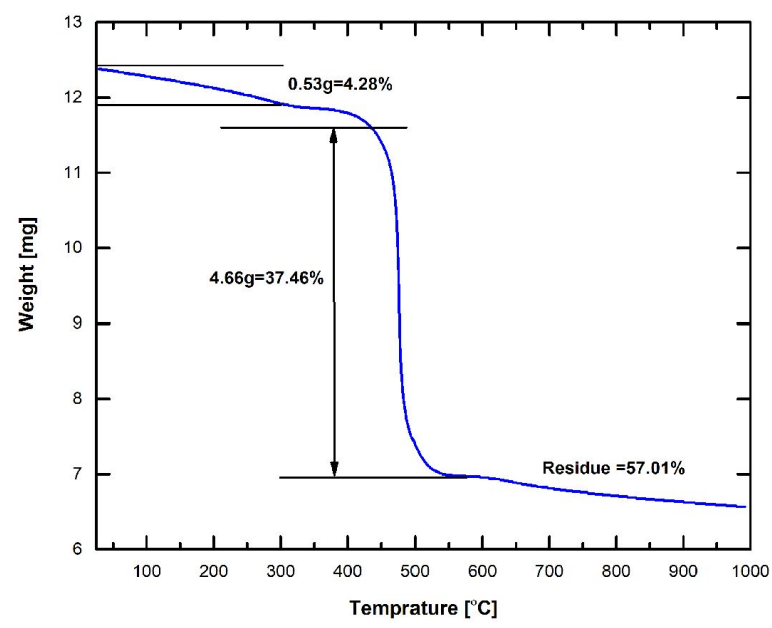

Fig. 5. TGA analysis of tin sulfide nanoparticles.

The weight of the sample was around $4.28 \%$ of initial material. On further heating from $400{ }^{\circ} \mathrm{C}$ to $500{ }^{\circ} \mathrm{C}$, the weight was decreased drastically probably due to the oxidizing of sulfur. About $57 \%$ of the material was left as a residue in the sample.

The melting point of $\mathrm{Sn}$ is quite low, around $231.9{ }^{\circ} \mathrm{C}$, on the other hand, the boiling point is very high i.e. $2602{ }^{\circ} \mathrm{C}$. Thus, the remaining residue in the sample is pure tin which was in amount about $55 \%$ in the starting material.

\subsection{Optical analysis}

The optical properties of the sample were characterized by diffuse reflectance spectroscopy. The spectra were measured in the range of $250 \mathrm{~nm}$ to $1000 \mathrm{~nm}$. The optical band gap of the nanoparticles was calculated using Kubelka-Munk function and Tauc plot:

$$
F\left(R_{\infty}\right)=\frac{(1-R)^{2}}{2 R}
$$




$$
(F(R) h v)^{1 / n}=B\left(h v-E_{g}\right)
$$

where $\mathrm{R}$ is the reflectance, $\mathrm{h}$ is Planck constant, $v$ is frequency of vibration, $\mathrm{B}$ is proportionality constant and $\mathrm{E}_{\mathrm{g}}$ is the energy bandgap $(\mathrm{eV})$. The values for $n$ are $1 / 2$ and 2 for direct allowed and indirect allowed transitions, respectively.

The obtained diffuse reflectance spectrum has been converted to Kubelka-Munk function $F(R)$. Thus, the vertical axis is converted to $F\left(R_{\infty}\right)$, which is proportional to the absorption coefficient $\alpha$. Using the Kubelka-Munk relation, $\left(\mathrm{h} v \cdot \mathrm{F}(\mathrm{R} \infty)^{\mathrm{n}}\right.$ was plotted as a function of energy. The curve of $\left(\mathrm{h} v-(\mathrm{h} v \cdot \mathrm{F}(\mathrm{R} \infty))^{2}\right)$ and the respective tangent on the horizontal axis $\mathrm{h} v$ and the vertical axis $(\mathrm{h} v \mathrm{~F}(\mathrm{R} \infty))^{2}$ have been drawn. The unit for $\mathrm{h} v$ is $\mathrm{eV}$, and its relationship to the wavelength $\lambda(\mathrm{nm})$ becomes $\mathrm{h} v=1239.7 / \lambda$.

A tangent has been drawn to the point of inflection and $h v$ value at the point of intersection with the horizontal axis, which defines the energy band gap $\mathrm{E}_{\mathrm{g}}$ value, has been determined. The direct band gap of $\mathrm{SnS}$ nanoparticles is $1.41 \mathrm{eV}$ as shown in Fig. 6.

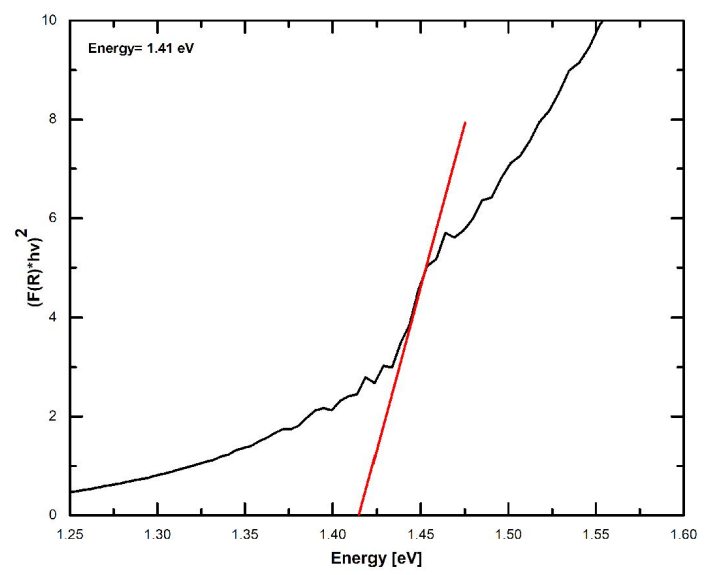

Fig. 6. Tauc plot for calculation of band gap of tin sulfide nanoparticles.

\subsection{Fourier transform infrared spec- troscopy FT-IR}

Fig. 7 shows the FT-IR spectra of $\mathrm{SnS}$ nanoparticles. The spectrum of the synthesized sample was recorded in the range from $4000 \mathrm{~cm}^{-1}$ to $400 \mathrm{~cm}^{-1}$. The intense broadband in the range of $3600 \mathrm{~cm}^{-1}$ to $4000 \mathrm{~cm}^{-1}$ is attributed to the stretching vibration of $\mathrm{O}-\mathrm{H}$ group, whereas the band at $1550 \mathrm{~cm}^{-1}$ to $1850 \mathrm{~cm}^{-1}$ correspond to bending vibrations of $\mathrm{O}-\mathrm{H}$ and $\mathrm{C}=\mathrm{O}$ groups. The band around $2330 \mathrm{~cm}^{-1}$ indicates presence of $\mathrm{CO}_{2}$ molecules at the surface. The bands at $1150 \mathrm{~cm}^{-1}$ to $1100 \mathrm{~cm}^{-1}$ correspond to $\mathrm{Sn}(\mathrm{OH})_{6}^{2-}$ vibrational modes. $\mathrm{S}_{2} \mathrm{O}_{6}{ }^{2-}$ vibration modes are identified at $1250 \mathrm{~cm}^{-1}$. The strongest peak at $930 \mathrm{~cm}^{-1}$ shows the formation of $\mathrm{Sn}-\mathrm{S}$ bond. The FT-IR spectra confirm the high purity of our sample except the oxygen bonding. The results of FT-IR analysis are in good agreement with XRD results.

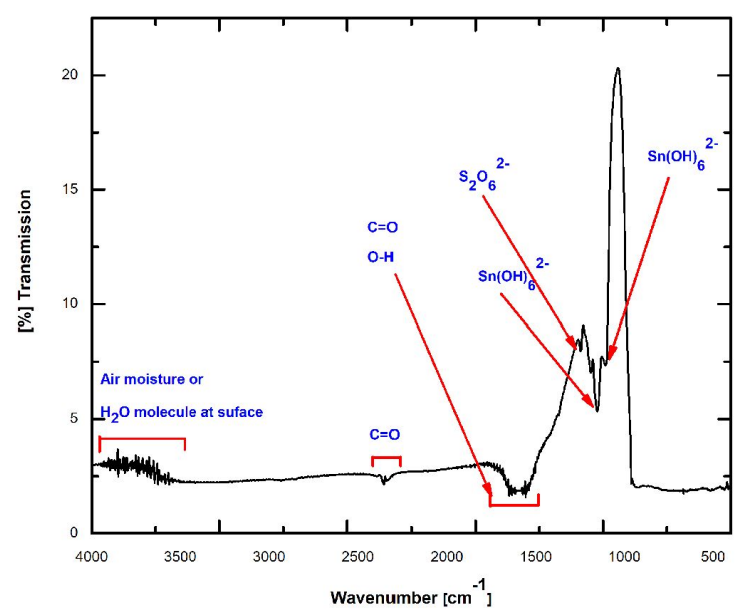

Fig. 7. FT-IR spectrum of tin sulfide nanoparticles.

\section{Conclusions}

Tin based chalcogenide nanoparticles were fabricated by co-precipitation method for next generation environmental friendly solar cell applications. The structural, morphological, optical and thermal properties were investigated using XRD, SEM, EDX, DRS, FT-IR and TGA. The XRD revealed orthorhombic phase of tin sulfide nanoparticles with average particle size of $22.1 \mathrm{~nm}$. The particle size of $50 \mathrm{~nm}$ was confirmed by SEM with $\pm 10 \mathrm{~nm}$ uncertainty. The elemental analysis by EDX showed that the content of tin and sulfur was 
$56.82 \mathrm{wt} . \%$ and $15.10 \mathrm{wt} . \%$, respectively. TGA was used to study physical and chemical changes in the sample as a function of temperature. We observed that with increasing temperature the weight of the sample was reduced. The presence of functional group was confirmed by FT-IR; the strong peak was observed for $\mathrm{SnS}$ at $930 \mathrm{~cm}^{-1}$. The optical bandgap of $1.41 \mathrm{eV}$ was evaluated using KubelkaMunk function and DRS. Such bandgap is favorable for photovoltaic devices.

\section{References}

[1] Zhirnov A., Karimova S., Ovsyannikova L., Gubenko O., Met. Sci. Heat Treat., 45 (2003), 23.

[2] Wakeham S., Hawkins G., Henderson G., Carthey N., Appl. Optics, 13 (2008), 206.

[3] Peralta G. L., Fontanos P. M., J. Mater. Cycles Waste, 1 (2006), 34.

[4] ZHaO Y., NALWA H.S., Nanotoxicology: interactions of nanomaterials with biological systems, American Scientific Publishers, California, 2007.

[5] Singh S., Nalwa H. S., J. Nanosci. Nanotechno., 9 (2007), 3048.

[6] Burton L.A., Colombara D., Abellon R.D., Grozema F.C., Peter L.M., SAVeniJe T.J., Dennler G., Walsh A., Chem. Mater, 24 (2013), 4908.

[7] Jomova K., Jenisova Z., Feszterova M., Baros S., Liska J., Hudecova D., Rhodes C., VAlko M., J. Appl. Toxicol., 2 (2011), 95.
[8] Rauch T., Boberl M., Tedde S.F., Furst J., KoVAlenko M.V., Hesser G., Lemmer U., Heiss W., Hayden O., Nat. Photonics, 6 (2009), 332.

[9] Reddy K.R., Reddy N.K., Miles R., Sol. Energ. Mat. Sol. C., 18 (2006), 3041.

[10] Albers W., HaAs C., Vink H., Wasscher J.D., $J$. Appl. Phys., 10 (1961), 2220.

[11] Blaesser G., Rossi E., Sol. Cell., 2 (1988), 91.

[12] Kawano K., Nakata R., Sumita M., J. Phys. D Appl. Phys., 1 (1989), 136.

[13] Ettema A., de Groot R., HaAs C., Turner T., Phys. Rev. B, 12 (1992), 7363.

[14] Yue G., Peng D., Yan P., Wang L., Wang W., LuO X., J. Alloy. Compd., 1 (2009), 254.

[15] Subramanian B., Sanjeeviraja C., JayachanDRAN M., Mater. Chem. Phys., 1 (2001), 40.

[16] Mahalingam T., John V., Rajendran S., SebasTIAN P., Semicond. Sci. Tech., 5 (2002), 465.

[17] Ortiz A., Alonso J., Garcia M., Toriz J., Semicond. Sci. Tech., 2 (1996), 243.

[18] Yue G., Wang W., Wang L., Wang X., Yan P., Chen Y. Peng D., J. Alloy. Compd., 1 (2009), 445.

[19] Devika M., Reddy N.K., RAMESH K., GUNASEKHAR K., Gopal E., REDdy K.R., J. Electrochem. Soc., 8 (2006), 727.

[20] Ullah H., Mari B., Superlattice. Microst., 2014, 148.

[21] Valmas A., Magiouf K., Fili S., Norrman M., Schluckebier G., Beckers D., Degen T., Wright J., Fitch A., Gozzo F., Acta Crystallogr. D, 4 (2015), 819.

Received 2017-07-15 Accepted 2018-03-17 